\title{
Caries-risk assessment in early childhood using a caries activity test
}

\author{
Michiko Nishimura $^{1}$, Omar M.M. Rodis ${ }^{2, *}$, Naoyuki Kariya ${ }^{1}$ and Seishi Matsumura ${ }^{2}$ \\ ${ }^{1}$ Pediatric Dentistry, Okayama University Hospital of Medicine and Dentistry \\ 2-5-1 Shikata-cho, Okayama 700-8525, JAPAN \\ ${ }^{2}$ Department of Behavioral Pediatric Dentistry, \\ Okayama University Graduate School of Medicine, Dentistry and Pharmaceutical Sciences \\ 2-5-1 Shikata-cho, Okayama 700-8525, JAPAN
}

\begin{abstract}
BACKGROUND: Caries-risk assessment based on the individual caries-risk is very important in the public health setting. The objective was to longitudinally assess the caries-risk of each group using cutoff points. Each groups were low, moderate, progressive border, improved border, moderately high, and high caries-risk groups which were determined based on the 18-month and 2-year-old current and 3.5-year-old predicted cutoff points. METHODS: This study was a two-year longitudinal study of 1,206 children born in 2000 . The children were divided into six caries-risk groups using the current and 3.5-year-old predicted cutoff points at 18 months and 2 years of age. It was investigated as to which caries-risk assessment was better. RESULTS: The 3.5-year-old predicted cutoff points calculated based on the 18-month and 2-year-old caries activities were more suitable for caries-risk assessment through analysis of significant differences of the mean numbers of $\mathrm{dft}$ in each groups. CONCLUSION: It was suggested that researchers and practitioners should try to keep caries-risk of children in the low or moderate caries-risk groups for caries prevention.
\end{abstract}

Key words

Caries activity test, Caries-risk assessment, Early childhood, Longitudinal study

\section{Introduction}

Despite the growing emphasis on targeting dental care through caries-risk assessment, little is known about the grouping of individuals according to their individual caries-risk. Dental caries is preventable and in order to devise a needs-related treatment plan for each patient, a proper caries-risk assessment tool based on scientific evidence is needed. Caries-risk in children should be assessed because it provides the health care professional with an estimate of future caries activity ${ }^{1)}$. The Cariostat test (DentsplySankin Co., Tokyo, Japan) is a colorimetric cariesrisk assessment test developed by Shimono ${ }^{2}$. The test medium contains $20 \%$ sucrose and two kinds of $\mathrm{pH}$ indicators to visually show the continuous

\footnotetext{
* Correspondence to: Omar M.M. Rodis

E-mail: omarodis@md.okayama-u.ac.jp

Received on December 28, 2010; Accepted on April 13, 2011
}

decrease of $\mathrm{pH}$ in the test medium caused by microorganisms in the patient's plaque sample. Some researchers have reported strong correlations between $\mathrm{pH}$ and the Cariostat score ${ }^{3-6}$. In a study by Tsubouchi, monitoring a child's caries activity test was useful in determining his or her caries-risk ${ }^{7}$. Newbrun reported that a good caries activity test should possess at least three characteristics: validity, reliability, and feasibility ${ }^{8}$. Snyder showed that an effective caries activity test should be based on appropriate theories, correlate closely with clinical status, be accurate concerning duplication of results, be simple, inexpensive, and requires little time ${ }^{9)}$. The caries activity test, Cariostat (Dentsply-Sankin Co., Tokyo), has the prerequisite conditions for a caries activity test as Snyder and Newbrun suggested. The Cariostat has become one of the popular tests in Japan for young patients ${ }^{10}$. Berg stated the Cariostat as a reliable and valid test ${ }^{11}$. A previous study has 
shown that the 18-month and 2-year-old Cariostat test results, except for Cariostat scores 0 and 3.0, and the 18-month-old Cariostat score of 0.5, not only reflected current caries status, but also predicted caries incidence at 2 and 3.5 years of age ${ }^{12}$. For improvement in the dental health of children, there is a need for an increased targeted prevention. In the public health setting where allocation of often scare resources is of prime importance, it is essential to understand how to classify children into different caries-risk groups. This may aid to utilize judiciously funds that are available for preventive services. In addition, the fact that individuals have unequal probabilities of caries indicates that appropriate management of caries will depend on accurate risk prediction ${ }^{13)}$. Therefore, accurate current and predicted caries-risk analysis based on scientific evidence in early childhood is necessary to establish the individual caries-risk and planning for suitable individual caries preventive treatment. There are reports regarding caries-risk grouping based on Cariostat test results ${ }^{3,7,14)}$. However, there is still no report about caries-risk assessment based on scientific evidence of Cariostat test results. The objective of this study was to investigate if the Cariostat could divide a population of 3.5-year-old children into six caries-risk groups: low and progressive border, moderate, improved border, moderately high, and high caries-risk in a longitudinal study to be able to establish individualized caries-risk assessment.

\section{Subjects and Methods}

The study population originally comprised of 1,208 children born in 2000 who participated in health examinations at 18 months, 2 years, and 3.5 years of age at Kurashiki City Public Health Center in Japan. Children taking any type of medication during any of their two Cariostat sampling periods were excluded from this study. Two patients were also excluded because they did not receive the Cariostat test at 18 months of age.

The oral examination system in Kurashiki City The 18-month and 3.5-year-old oral examinations are routine Japanese-sanctioned national dental check-up activities while the 2-year-old oral examination is Kurashiki City's local dental check-up activity, as part of their community's general health program. These monthly programs began in 1975, 1961, and 1980, respectively. Caries-risk assessment was done based on Cariostat test results. For this study, two pediatric dentists from Okayama University carried out Cariostat testing at 18 months and 2 years of age while dentists of Kurashiki City Dental Society carried out the examinations during the 18-month and 3.5-year-old oral examination. Oral examinations during the 2-year-old oral examination were done by the same pediatric dentists from Okayama University. Calibration of the oral examination was not possible because of the different programs. However, these examinations have been done regularly by the same dentists and in accordance with the criteria of the Health Policy Bureau, Ministry of Health and Welfare, Japan ${ }^{15}$. Local city health officers completely sealed each of the subjects' private information and released the data to us in coded form.

\section{Cariostat sampling}

Plaque samples were collected from the maxillary buccal cervical surfaces using a sterile cotton swab. The swab was rubbed along the tooth surfaces five times in a wiping motion prior to placing it into the ampoule containing $2 \mathrm{ml}$ of Cariostat test medium. This medium was incubated at $37^{\circ} \mathrm{C}$ for 48 hours. After incubation, a score was assigned with reference to four standard colors. Each of these scores is evaluated as follows; score $0(\mathrm{pH} 5.8-7.2), 1.0$ $(\mathrm{pH} 5.4 \pm 0.3), 2.0(\mathrm{pH} 4.8 \pm 0.3)$, and $3.0(\mathrm{pH}<4.4)$. Public dental hygienists of Kurashiki City evaluated the test results using the four reference colors included in the Cariostat kit. In this study, the modified 7-scale was used in which the interval of $0-1.0,1.0-2.0$, and 2.0-3.0 were included. The test results were also sent to individual participants by postal mail.

Current and predicted cutoff points

The 18-month and 2-year-old current cutoff points were calculated using discriminate analysis and the gold standards were caries-free or caries-experience at either ages. The 3.5-year-old predicted cutoff points based on the 18-month and 2-year-old test results were calculated based on both age's scores using discriminate analysis and the gold standard was caries-free or experience at 3.5 years of age. The current and the 3.5-year-old predicted points based on the 18-month and 2-year-old test results that had the highest validities were adopted as cutoff points for caries-risk assessment. The current cutoff points at 18 months and 2 years of age were 1.0 


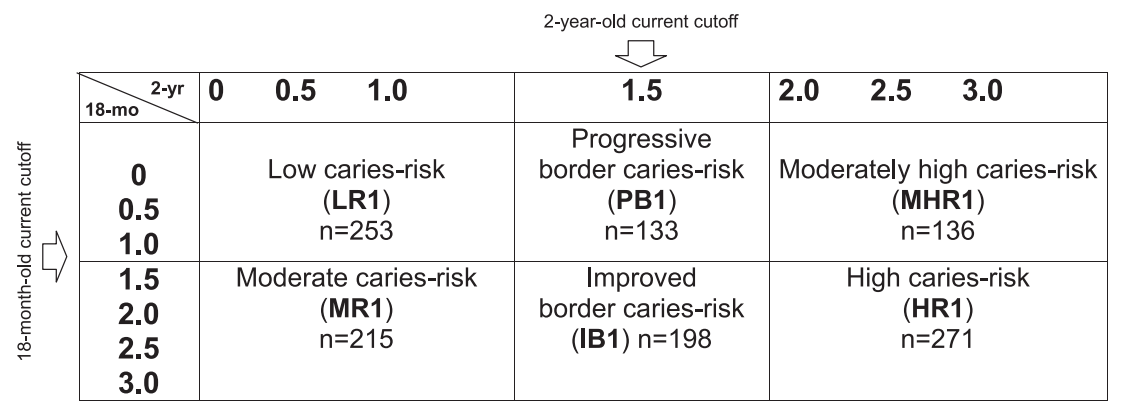

Fig. 1 Caries-risk assessment based on the 18-month and 2-year-old current cutoff points

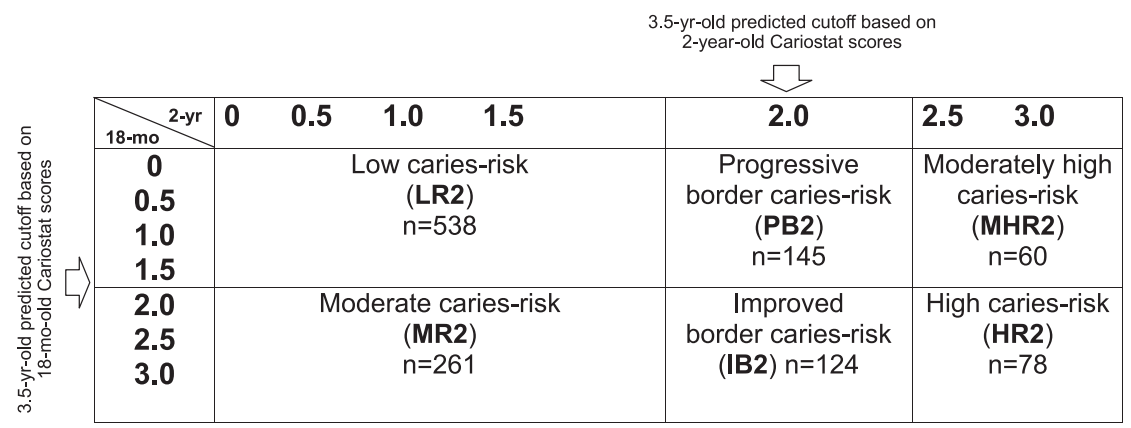

Fig. 2 The 3.5-yr-old predicted caries-risk assessment based on the 18-month and 2-year-old current cutoff points

and 1.5 , respectively and the 3.5 -year-old predicted cutoff points based on the 18-month and 2-year-old test results were 1.5 and 2.0 , respectively.

Caries-risk assessment according to caries-risk The 18-month and 2-year-old current cutoff points and 3.5-year-old predicted cutoff points based on the 18-month-old and 2-year-old Cariostat test results that had the highest validities were used for cariesrisk assessment. As a result, patients were divided into six groups. Then, the current cutoff points or the 3.5-year-old predicted cutoff points was checked as to which was better for caries-risk assessment for the 2-year-old and 3.5-year-old children. Patients were divided into six caries-risk groups: low, moderate, progressive border, improved border, moderately high and high caries-risk groups. These groupings were based on the 18-month and 2-year-old current cutoff points (Cariostat score, 1.0 and 1.5, respectively) and the 3.5-year-old predicted cutoff points based on 18-month and 2-year-old Cariostat test results (Cariostat score, 1.5 and 2.0, respectively). The six caries-risk groups are as follows; (1) Low caries-risk group (LR): children who had a Cariostat score at or lower than the 18-month-old cutoff point, and a score lower than the 2-year-old cutoff point. (2) Moderate caries-risk group (MR): children who had a Cariostat score higher than the 18-month-old cutoff point but had scores lower than the 2-year-old cutoff point. (3) Progressive border caries-risk group (PB): children who had a Cariostat score at or lower than the 18-month-old cutoff point but reached the 2-year-old cutoff point. (4) Improved border cariesrisk group (IB): children who had a Cariostat score higher than the 18-month-old cutoff point but reached the 2-year-old cutoff point. (5) Moderately high caries-risk group (MHR): children who had a Cariostat score at or lower than the 18-month-old cutoff point but had higher scores than the 2-yearold cutoff point. (6) High caries-risk group (HR): children who had higher Cariostat scores than the cutoff points at both ages. Caries-risk grouping based on the 18-month and the 2-year-old current cutoff points is shown in Fig. 1. Caries-risk grouping of the 3.5-year-old predicted cutoff points based on the 18-month and the 2-year-old Cariostat scores is shown in Fig. 2. 


\begin{tabular}{|l|c|c|c|c|c|c|}
\hline & LR1 & MR1 & PB1 & IB1 & MHR1 & HR1 \\
\hline LR1 & & $* *$ & NS & ${ }^{* *}$ & ${ }^{* * *}$ & ${ }^{* * *}$ \\
\hline MR1 & & & NS & NS & NS & ${ }^{* * *}$ \\
\hline PB1 & & & & NS & ${ }^{* *}$ & ${ }^{* * *}$ \\
\hline IB1 & & & & & $*$ & $* * *$ \\
\hline MHR1 & & & & & & $*$ \\
\hline HR1 & & & & & & \\
\hline
\end{tabular}

The significant differences of the mean numbers of decayed and filled teeth ${ }^{*} P<0.05 \quad{ }^{*} P<0.01 \quad{ }^{* * *} P<0.001 \quad \mathrm{NS}=$ not significant

\begin{tabular}{|c|c|c|c|}
\hline & $\begin{array}{lll}0 & 0.5 & 1.0 \\
\end{array}$ & 1.5 & $\begin{array}{lll}2.0 & 2.5 & 3.0\end{array}$ \\
\hline $\begin{array}{c}0 \\
0.5 \\
1.0\end{array}$ & $\begin{array}{c}\text { Low caries-risk } \\
\text { (LR1) } \\
n=253\end{array}$ & $\begin{array}{c}\text { Progressive } \\
\text { border } \\
\text { caries-risk } \\
\text { (PB1) } \\
n=133\end{array}$ & $\begin{array}{c}\text { Moderately high } \\
\text { caries-risk } \\
\text { (MHR1) } \\
n=136\end{array}$ \\
\hline $\begin{array}{l}1.5 \\
2.0 \\
2.5 \\
3.0\end{array}$ & $\begin{array}{c}\text { Moderate caries- } \\
\text { risk } \\
\text { (MR1) } \\
n=215\end{array}$ & $\begin{array}{l}\text { Improved } \\
\text { border } \\
\text { caries-risk } \\
\text { (IB1) } n=198\end{array}$ & $\begin{array}{l}\text { High caries-risk } \\
\begin{array}{c}\text { (HR1) } \\
n=271\end{array}\end{array}$ \\
\hline
\end{tabular}

Risk distribution between the six caries-risk groupings

$$
\text { 2-year-old children }
$$

\begin{tabular}{|l|c|c|c|c|c|c|}
\hline & LR1 & MR1 & PB1 & IB1 & MHR1 & HR1 \\
\hline LR1 & & NS & NS & $*$ & $* *$ & $* * *$ \\
\hline MR1 & & & NS & NS & NS & ${ }^{* * *}$ \\
\hline PB1 & & & & NS & NS & ${ }^{* * *}$ \\
\hline IB1 & & & & & NS & ${ }^{* * *}$ \\
\hline MHR1 & & & & & & ${ }^{* * *}$ \\
\hline HR1 & & & & & & \\
\hline
\end{tabular}

The significant differences of the mean numbers of decayed and filled teeth ${ }^{*} P<0.05 \quad{ }^{* *} P<0.01 \quad{ }^{* * *} P<0.001 \quad N S=$ not significant

\begin{tabular}{|c|c|c|c|}
\hline & $\begin{array}{lll}0 & 0.5 & 1.0\end{array}$ & 1.5 & $\begin{array}{lll}2.0 & 2.5 & 3.0\end{array}$ \\
\hline $\begin{array}{l}0 \\
0.5 \\
1.0\end{array}$ & $\begin{array}{c}\text { Low caries-risk } \\
\text { (LR1) } \\
n=253\end{array}$ & $\begin{array}{c}\text { Progressive } \\
\text { border } \\
\text { caries-risk } \\
\text { (PB1) } \\
n=133\end{array}$ & $\begin{array}{c}\text { Moderately high } \\
\text { caries-risk } \\
\text { (MHR1) } \\
n=136\end{array}$ \\
\hline $\begin{array}{l}1.5 \\
2.0 \\
2.5 \\
3.0\end{array}$ & $\begin{array}{c}\text { Moderate caries- } \\
\text { risk } \\
\text { (MR1) } \\
n=215\end{array}$ & $\begin{array}{l}\text { Improved } \\
\text { border } \\
\text { caries-risk } \\
\text { (IB1) } n=198\end{array}$ & $\begin{array}{l}\text { High caries-risk } \\
\qquad \begin{array}{c}\text { (HR1) } \\
n=271\end{array}\end{array}$ \\
\hline
\end{tabular}

Risk distribution between the six caries-risk groupings

\section{5-year-old children}

Fig. 3 The significant differences in the mean number of decayed and filled teeth based on the 2-year-old current cutoff point

\begin{tabular}{|l|c|c|c|c|c|c|}
\hline & LR2 & MR2 & PB2 & IB2 & MHR1 & HR2 \\
\hline LR2 & & $* *$ & NS & ${ }^{* *}$ & ${ }^{* * *}$ & ${ }^{* * *}$ \\
\hline MR2 & & & ${ }^{* * *}$ & ${ }^{* * *}$ & ${ }^{* * *}$ & ${ }^{* * *}$ \\
\hline PB2 & & & & ${ }^{* * *}$ & ${ }^{* *}$ & $* * *$ \\
\hline IB2 & & & & & NS & NS \\
\hline MHR2 & & & & & & NS \\
\hline HR2 & & & & & & \\
\hline
\end{tabular}

The significant differences of the mean numbers of decayed and filled teeth ${ }^{*} P<0.05 \quad{ }^{* *} P<0.01 \quad{ }^{* * *} P<0.001 \quad$ NS $=$ not significant

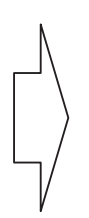

\begin{tabular}{|l|c|c|c|c|c|c|}
\hline & LR2 & MR2 & PB2 & IB2 & MHR2 & HR2 \\
\hline LR2 & & $* * *$ & NS & $* * *$ & $* * *$ & $* * *$ \\
\hline MR2 & & & NS & $* * *$ & $* * *$ & $* * *$ \\
\hline PB2 & & & & $* * *$ & $* *$ & $* * *$ \\
\hline IB2 & & & & & NS & ${ }^{* * *}$ \\
\hline MHR2 & & & & & & $* * *$ \\
\hline HR2 & & & & & & \\
\hline
\end{tabular}

The significant differences of the mean numbers of decayed and filled teeth ${ }^{*} P<0.05 \quad{ }^{* *} P<0.01 \quad{ }^{* * *} P<0.001 \quad$ NS $=$ not significant

\begin{tabular}{|c|c|c|c|}
\hline & $\begin{array}{lll}0 & 0.5 & 1.0 \\
\end{array}$ & 1.5 & $\begin{array}{lll}2.0 & 2.5 & 3.0\end{array}$ \\
\hline $\begin{array}{c}0 \\
0.5 \\
1.0\end{array}$ & $\begin{array}{c}\text { Low caries-risk } \\
\text { (LR2) } \\
n=538\end{array}$ & $\begin{array}{c}\text { Progressive } \\
\text { border } \\
\text { caries-risk } \\
\text { (PB2) } \\
n=145\end{array}$ & $\begin{array}{c}\text { Moderately high } \\
\text { caries-risk } \\
\text { (MHR2) } \\
n=60\end{array}$ \\
\hline $\begin{array}{l}1.5 \\
2.0 \\
2.5 \\
3.0\end{array}$ & $\begin{array}{c}\text { Moderate caries- } \\
\text { risk } \\
\text { (MR1) } \\
n=261\end{array}$ & $\begin{array}{c}\text { Improved } \\
\text { border } \\
\text { caries-risk } \\
\text { (IB2) } n=124\end{array}$ & $\begin{array}{l}\text { High caries-risk } \\
\qquad \begin{array}{c}\text { (HR2) } \\
n=78\end{array}\end{array}$ \\
\hline
\end{tabular}

Risk distribution between the six caries-risk groupings

\section{5-year-old children}

Fig. 4 The significant differences in the mean number of decayed and filled teeth based on the 3.5-year-old predicted cutoff point 
Table 1 The 2- and 3.5-year-old caries status of each caries-risk groups based on the 3.5-year-old predicted cutoff points

\begin{tabular}{ccccccc}
\hline Caries-risk group & LR 2 & MR 2 & PB 2 & IB 2 & MHR 2 & HR 2 \\
\hline 2-year-old caries status & $0.09(0.43)$ & $0.20(0.82)$ & $0.07(0.35)$ & $0.53(1.25)$ & $0.53(1.41)$ & $0.78(1.57)$ \\
3.5-year-old children & $0.77(1.85)$ & $1.11(2.47)$ & $0.90(1.96)$ & $2.44(3.50)$ & $2.03(2.83)$ & $4.14(4.63)$ \\
$\mathrm{n}$ & 538 & 261 & 145 & 124 & 60 & 78 \\
\hline
\end{tabular}

Table 2 The 2- and 3.5-year-old caries prevalence of each caries-risk groups based on the 3.5-year-old predicted cutoff points

\begin{tabular}{ccccccc}
\hline Caries-risk group & LR 2 & MR 2 & PB 2 & IB 2 & MHR 2 & HR 2 \\
\hline 2-year-old caries status & 4.09 & 7.66 & 4.14 & 14.8 & 18.3 & $29.5(\%)$ \\
3.5-year-old children & 24.2 & 31.8 & 33.8 & 48.4 & 46.7 & $62.8(\%)$ \\
$\mathrm{n}$ & 538 & 261 & 145 & 124 & 60 & 78 \\
\hline
\end{tabular}

\section{Statistical methods}

All analyses were performed using SPSS (Statistical Package for the Social Sciences) Ver. 12.5. The significant differences among the mean decayed and filled numbers of teeth in each of the groups were analyzed using Mann-Whitney $U$ test. A $P$-value below 0.05 was considered significant.

\section{Results}

\section{Caries-risk assessment}

Based on the 18-month and 2-year-old current cutoff points

Figure 1 shows the caries-risk assessment groupings based on the 18-month and 2-year-old current cutoff points. These cutoff points could divide caries risk of children into seven caries-risk groups; $\underline{L R} 1$ and

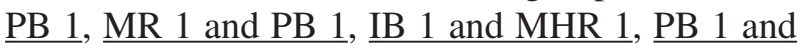
$\underline{\text { IB } 1}, \underline{\text { IB } 1,}$ MHR 1 , and $\underline{\text { HR } 1}$ at 2 years of age and could divide the same children into six caries-risk groups; LR 1, MR 1, and PB 1, MR 1, PB 1, IB 1, and MHR 1, PB 1, IB 1, and MHR 1, IB 1 and MHR 1 , MHR 1, and HR 1 (underline shows the same risk group) at age of 3.5 years presented as riskdistribution (Fig. 3). Based on the shading, the caries-risk assessment of the progressive border caries-risk group at 2 years old and the improved border and moderately high caries-risk groups at 3.5 years old were not clearly distributed.

Based on the 3.5-year-old predicted cutoff points Figure 4 shows the caries-risk assessment based on the 3.5-year-old predicted cutoff points. These cutoff points could divide the children into five caries-risk groups; LR 2 and PB 2, MR 2, $\underline{\text { PB 2 }}, \underline{\mathrm{IB}}$ 2, MHR 2, and HR 2, and MHR 2 and HR 2 at 2 years of age and could divide the same children, into six caries-risk groups; LR 2 and PB 2, MR 2 and PB 2, PB 2, IB 2 and MHR 2, MHR 2, and HR 2 at age of 3.5 years presented as risk-distribution. The riskdistribution based on 3.5-year-old predicted cutoff points more clearly showed caries-risk assessment than the caries-risk assessment based on current cutoff points at age of 18 months and 2 years.

Caries status of each group at 2 and 3.5 years of age Table 1 shows the mean df number of each group based on the 3.5-year-old predicted cutoff point at both ages while Table 2 shows caries prevalence.

\section{Discussion}

Koch reported the importance of the determination of caries-risk ${ }^{16)}$. The American Academy of Pediatric Dentistry (AAPD) reported that caries-risk assessment is an essential element of contemporary clinical care for infants and children ${ }^{17}$. Furthermore, strategies for managing dental caries have increasingly emphasized the concept of risk-assessment. However, practical tools for assessing caries in infants and children have been lacking ${ }^{18}$. The Cariostat is very simple, safe, requires short sampling time and shows excellent responsibility and predictive abilities 3,5-7,12,14) $_{\text {. }}$ In selected reports ${ }^{3,7,17)}$, patients were classified into two or three groups: Low-risk and high caries-risk 
groups or low, moderate, and high caries-risk groups in a cross-sectional and a longitudinal study.

In a previous study ${ }^{12)}$, the current and 3.5-yearold predicted screening indices were calculated through discriminate analysis. In the present study, original and scientifically proven caries-risk assessment method was suggested. Caries-risk assessments were performed based on the 18-month and 2-yearold cutoff points and the 3.5-year-old predicted cutoff points calculated based on the 18-month and 2 -year-old Cariostat test results. The present study was a challenge for caries-risk assessment performed based on individual oral conditions. Sensitivity is higher as the cutoff point is lower and specificity is higher as the cutoff point is higher. Therefore, the Cariostat test results that had the highest validities (sensitivity + specificity) were selected as the cutoff points in this study. It is useful to show the screening indices regarding every score to fix cutoff points for clinicians and researchers. There was no significant difference between low (LR) and progressive border (PB) caries-risk groups because the Cariostat test results of the patients in both groups were current or 3.5-year-old predicted cutoff points or below. If there was a significant difference, the current cutoff points or 3.5-year-old predicted cutoff points are insignificant. Furthermore, it is ideal that each group, except for LR and PB, to have significant differences between each other. Therefore, the 2-year-old and 3.5-year-old children's risk-assessment based on 3.5-year-old predicted cutoff points were better than caries-risk assessment based on the current cutoff points (Figs. 3 and 4).

Tables 1 and 2 show the mean number of decayed and filled teeth and caries prevalence of each cariesrisk groups based on 3.5-year-old cutoff points, respectively. The mean number of $\mathrm{df}$ and caries prevalence of the 3.5-year-old children in Kurashiki City were 1.32 and $32.4 \%$, respectively. Patients in LR 2, PB 2 and MR 2 had smaller df than the mean numbers, $(<1.32)$ and the caries prevalence in $\mathrm{LR}$ 2 and MR 2 were below 32.4\%. Researchers and clinicians will be able to improve the individual oral conditions and caries prevalence in the public health setting through adequate caries-risk assessment and they should fix the cutoff points to manage patients. Furthermore, these findings emphasized on the importance of the following oral managements for patients, clinicians, and researchers: to manage the patient's oral condition by maintaining a score of 1.5 or below at 18 months of age and to dramatically improve oral conditions below the score of 1.5 at 2 years of age if a patient's score is beyond 2.0 at 18 months of age. Dentists should manage their patients to be in LR 2 and MR 2. The predicted cutoff points that had the highest validities should be adopted because caries-risk assessment was effective from 18 months old to 3.5 years old through predicted cutoff points. Furthermore, these results agree with keeping a patient's caries activity as low as possible before the start of Caufield's window of infectivity (from 19 to 31 months old) ${ }^{19}$, which is a key factor for caries prevention in young children. Another key factor is to dramatically improve oral conditions below the cutoff point during Caufield's window of infectivity. Thus predictability of caries activity test may be the most important condition for caries-risk assessment. To be able to accurately predict a young child's caries-risk, we should at first investigate the sampling age, then determine the cutoff points, and recommend the score with the highest validity. Authors also consider that this caries-risk assessment methodology to predict future caries status for caries-risk assessment can be applied even with the use of other caries activity.

\section{Conclusion}

The predicted cutoff point that had the highest validity was adequate for caries-risk assessment. In summary, it is best to adopt the predicted cutoff points to be able to predict future caries status for caries-risk assessment. As a result, practitioners can offer an individual patient a needs-related caries preventive treatment. Researchers and practitioners should be able to manage patients to be within the low or moderate caries-risk groups to improve their oral health.

\section{References}

1) Saemundsson, S.R., Slade, G.D., Spencer, A.J. and Davies, M.J.: The basis for clinicians' caries risk grouping in children. Pediatr Dent 19: 331-338, 1997.

2) Shimono, T. and Sobue, S.: A new colorimetric method for caries diagnosis. Dental Outlook 42 (6): 829-835, 1974.

3) Sutadi, H., Jen, C.H., Nishimura, M., Matsumura, S. and Shimono, T.: The determination of the predictive value of caries activity test and its suitability for mass screening in Indonesia. Ped Dent J 2(1): 73-81, 1992.

4) Camling, E. and Emilson, C.G.: Results with the 
caries activity test "Cariostat" compared to prevalence of mutans streptococci and lactobacilli. Scand J Dent Res 14: 125-130, 1989.

5) Olofernes-Docor, R.: A longitudinal study of approximal caries in primary molars - Predictive value of Cariostat —. Ped Dent J 6 (1): 125-134, 1996.

6) Chen, H.J., Nishimura, M., Matsumura, S. and Shimono, T.: Comparison of mutans streptococci count methods and Cariostat test for caries risk assessment. Ped Dent J 5 (1): 31-42, 1995.

7) Tsubouchi, J., Yamamoto, S., Shimono, T. and Domoto, P.K.: A longitudinal assessment of predictive value of caries activity test in young children. ASDC J Dent Child 62 (1): 34-37, 1995.

8) Newbrun, E., Matsukubo, T., Hoover, R.C.G. and Bohannan, H.M.: Comparison of screening test for Streptococcus mutans and evaluation of their suitability for mass screening and private practice. Community Dent Oral Epidemiol 12: 325-331, 1984.

9) Snyder, M.L.: Laboratory method in the clinical evaluation of caries activity. J Am Dent Assoc 42: 400-413, 1951.

10) Kurokawa, I. and Shimokawa, S.: The caries activity test in primary dentition period for estimation of progression on the same subjects. Jpn J Ped Dent 29: 719-813, 1991.

11) Berg, H.B.: The marketplace for new caries management products: Dental caries detection and caries management by risk assessment. BMC Oral Health 6 (Suppl 1): 1-6, 2006.

12) Nishimura, M., Oda, T., Kariya, N., Matsumura, S. and Shimono, T.: Using caries activity test, Cariostat, to predict early childhood caries-risk. J Am Dent Assoc 139: 63-71, 2008.

13) Reich, E., Lussi, A. and Newbrun, E.: Caries-risk assessment. Int Dent J 49 (1): 15-26, 1990.

14) Koroluk, L., Hoover, J.N. and Komiyama, K.: The sensitivity and specificity of a colorimetric microbiological caries activity test (Cariostat) in preschool children. Pediatr Dent 16(4): 276-281, 1995.

15) Report on the survey of dental diseases by Health Policy Bureau, Ministry of Health and Welfare Japan. Oral Health Assoc Japan, Tokyo, pp.32-53.

16) Koch, G.: Importance of early determination of caries risk. Int Dent J 38: 203-210, 1988.

17) AAPD.: Policy on use of a caries-risk assessment tool (CAT) for infants, children, and adolescents. Pediatr Dent 24 (7): 15-17, 2004.

18) AAPD.: Guideline on periodicity of examination, preventive dental services, anticipatory guidance and oral treatment for children. Pediatr Dent 24(7): 51-52, 2002.

19) Caufield, P.W., Cutter, G.R. and Dasanayake, A.P.: Initial acquisition of mutans streptococci by infants: evidence for a discrete window of infectivity. $J$ Dent Res 72: 37-45, 1993. 